\title{
Synthesis of Vinyl- and Allylphosphonates by Olefin Cross-Metathesis
}

\author{
Arnab K. Chatterjee, Tae-Lim Choi, Robert H. Grubbs* \\ Arnold and Mabel Beckman Laboratories of Chemical Synthesis, Division of Chemistry and Chemical Engineering, California Institute of \\ Technology, Pasadena, California 91125, USA \\ E-mail: rhg@ caltech.edu \\ Received 15 February 2001
}

\begin{abstract}
Substituted allyl and vinyl phosphonates have been prepared for the first time via intermolecular olefin cross-metathesis (CM) using 1,3-dimesityl-4,5-dihydro-imidazol-2-ylidene ruthenium alkylidene complex $\mathbf{3}$ in good yield. A variety of terminal olefins, styrenes, and geminally disubstituted olefins have been successfully employed in these reactions. In addition, $\mathrm{CM}$ of vinylphosphonates provide exclusive $E$ olefin stereochemistry.
\end{abstract}

Key words: metathesis, ruthenium, phosphorus, cross-coupling, coupling

Olefins that contain phosphonate functionality are used extensively in synthetic organic chemistry. For example, allylic phosphonates are employed in the preparation of dienes and polyenes by Horner-Emmons olefination, providing products with improved stereoselectivity as compared to the corresponding phosphonium salts. ${ }^{1}$ The reaction of organic halides with trialkyl phosphites (Michaelis-Arbuzov reaction) is used primarily for the synthesis of allylphosphonates. ${ }^{2}$ However, elimination and/or loss of olefin stereochemical integrity are often competitive with product formation under the reaction conditions. Palladium-catalyzed cross-coupling of hydrogen phosphonates to conjugated dienes and allenes has also been developed, but requires high reaction temperatures and provides low regioselectivity in highly substituted phosphonate products. ${ }^{3}$

Vinylphosphonates are important synthetic intermediates ${ }^{4}$ and have been investigated as biologically active compounds. ${ }^{5}$ Vinylphosphonates ${ }^{6}$ have been used as intermediates in stereoselective synthesis of trisubstituted olefins ${ }^{7}$ and in heterocycle synthesis. ${ }^{8}$ The synthesis of vinylphosphonates has also been widely examined and a variety of non-catalytic approaches have been described in the literature. ${ }^{9}$ Recent metal-catalyzed methods include palladium-catalyzed cross-coupling ${ }^{10}$ and Heck coupling of aryldiazonium salts with vinyl phosphonates, ${ }^{11}$ but are limited by the requirement of highly reactive functional groups in the substrates. Therefore, a more mild, general and stereoselective method for the synthesis of vinyl- and allylphosphonates using commercially available starting materials would be valuable, and may provide an additional degree of orthogonality to the previously reported syntheses. In this letter, the productive cross-metathesis (CM) of allyl and vinylphosphonates with a variety of terminal olefins in excellent yields and good stereoselectivities is described. ${ }^{12}$

The olefin metathesis reaction ${ }^{13}$ has become important in organic synthesis with the commercial availability of well-defined transition metal catalysts, such as the molybdenum alkoxy-imido alkylidene $\mathbf{1}^{14}$ and ruthenium benzylidene 2. ${ }^{15}$ The preparation of olefins with vinylic functionality by olefin cross-metathesis ${ }^{16}$ has recently been demonstrated using the highly active rutheniumbased olefin metathesis catalyst $\mathbf{3}$, containing a 1,3-dimesityl-4,5-dihydro-imidazol-2-ylidene ligand. ${ }^{17}$ The homologation of olefins with a variety $\alpha, \beta$-unsaturated carbonyl compounds, including esters, ketones, aldehydes, and amides with high $(E)$-stereoselectivity has been previously described. ${ }^{18}$ In general, the catalyst $\mathbf{3}$ has also shown enhanced activity in ring-closing metathesis $(\mathrm{RCM})^{19}$ and ring-opening metathesis polymerization (ROMP). ${ }^{20}$ Phosphorus-containing $\alpha, \omega$-dienes, such as allylphosphonates and allylphosphoramides, have been utilized as RCM substrates by Hanson and co-workers using 2. ${ }^{21}$ In addition, Gouverneur and co-workers have demonstrated the intramolecular RCM of allylic phosphine oxides, phosphinates, and phosphoboranes using $\mathbf{2}$ and an unsaturated analog of $\mathbf{3}^{22}$ However, the intermolecular $\mathrm{CM}$ reactions of phosphonates have not been previously reported.

Olefins couple efficiently with diethylvinylphosphonate to generate $\alpha, \beta$-unsaturated phosphonates in excellent yield using catalyst 3 . Terminal olefins were reacted with commercially available diethyl vinylphosphonate and after column chromatography, a $95 \%$ yield of CM product was obtained exclusively as the $E$-isomer (Table 1, Entry 1). Importantly, no dimerization of the vinylphosphonate

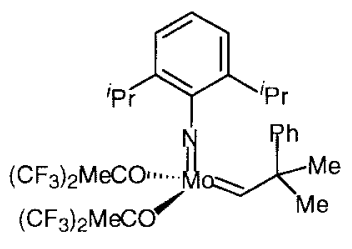

1

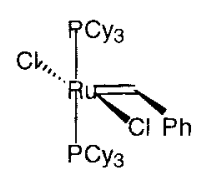

2

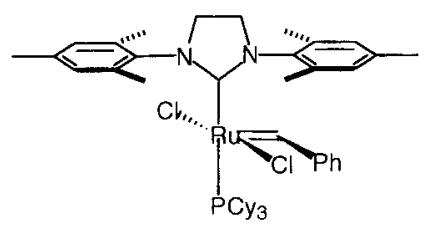

3 
Table 1 Synthesis of Vinylphosphonates by Olefin Cross-Metathesis

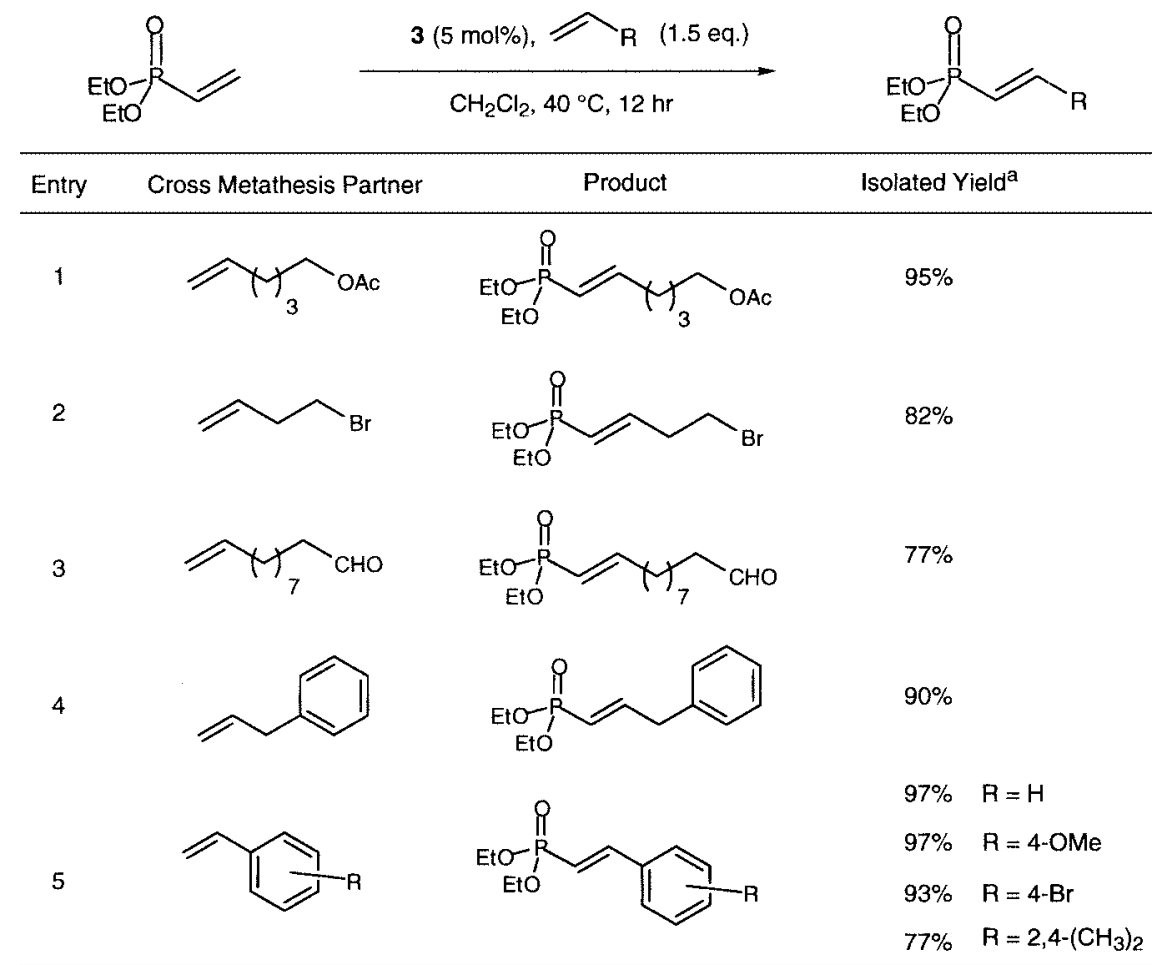

a $>$ 20:1 E/Z as determined by ${ }^{1} \mathrm{H}$ NMR.

was detected by ${ }^{1} \mathrm{H}$ NMR allowing for selective CM. Alkyl halide (Entry 2) and unprotected aldehyde functionalities (Entry 3 ) are well tolerated with the ruthenium catalyst 3 . These reactive functional groups are generally used in allyl and vinylphosphonate synthesis, and CM provides a unique method to synthesize these compounds directly from olefins. Allyl benzene is an excellent partner for CM without olefin isomerization (Entry 4). Olefin isomerization of vinylphosphonates under basic reaction conditions has been previously described. ${ }^{23}$ The participation of a variety of styrenes in the $\mathrm{CM}$ reaction with vinylphosphonates is also interesting to note. These results indicate a variety of styrenes are converted to $(E)$-cinnamylphosphonates in excellent yield (Table 1, Entry 5). The CM method tolerates electronic and steric constraints in the styrene partner and allows for CM between two electron-deficient olefins. ${ }^{24}$

Second, diethylallylphosphonate was investigated as a potential CM partner. It has been previously demonstrated that some allylic functional groups improve cross-coupling and disfavor homodimerization by creating an electronic mismatch. ${ }^{16}$ As summarized in Table 2, allylphosponates are viable CM partners, providing slightly enhanced CM ratios relative to the predicted statistical mixture. In addition, some challenging styrenes are excellent CM partners (Table 2, Entry 2 and 3) and provide the $E$-isomer exclusively. A trisubstituted olefin is also formed in excellent yield with modest stereoselectivity (Table 2, Entry 6). All of the reaction products were easily separated from their respective homodimers by column chromatography.

In conclusion, the cross-metathesis of a variety of terminal olefins with allyl and vinylphosphonates employing the ruthenium alkylidene $\mathbf{3}$ has been described. These findings further demonstrate the functional group tolerance of 3, expanding the range of olefins that can participate in the olefin metathesis reaction, and provide an efficient method to prepare vinyl and allylphosphonates in high yield and good stereoselectivity.

\section{Acknowledgement}

Support has been generously provided by the National Institutes of Health. The authors thank Steven D. Goldberg for useful discussions and encouragement.

\section{References and Notes}

(1) For example: Crombie, L.; Hemesley, P.; Pattenden, J. J. Chem. Soc., Chem. Commun. 1969, 1024. Asato, A. E.; Mead, D.; Denny, M.; Bopp, T. T.; Liu, R. S. H. J. Am. Chem. Soc. 1982, 104, 4979. Maryanoff, B. E.; Reitz, A. B. Chem. Rev. 1989, 89, 863. Whang, K.; Venkataraman, H.; Kim, Y.; Cha, J. K. J. Org. Chem. 1991, 56, 7177.

(2) Bhattacharya, A. K.; Thyagarjan, G. Chem. Rev. 1981, 81, 415.

(3) Hirao, T.; Masunaga, T.; Oshiro, Y.; Agawa, T. Tetrahedron Lett. 1980, 21, 3595. Hirao, T.; Masunaga, T.; Yamada, N.; Oshiro, Y.; Agawa, T. Bull. Chem. Soc. Jpn. 1982, 55, 909. Imamoto, T.; Oshiki, T.; Onozawa, T.; Kusumoto, T.; Sato, K. 
Table 2 Synthesis of Allylphosphonates by CM

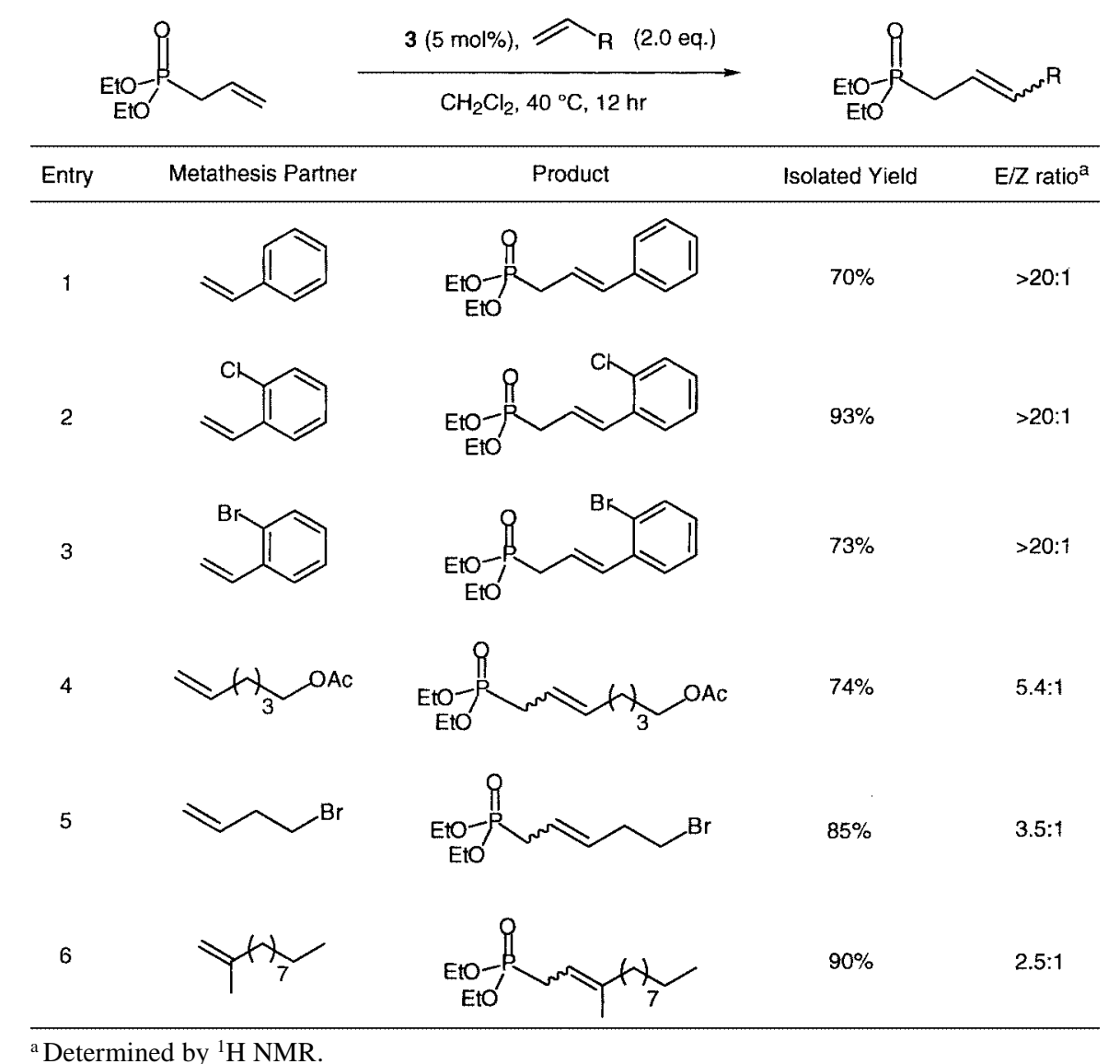

J. Am. Chem. Soc. 1990, 112, 5244. Zhao, C.-Q.; Han, L.-B.; Tanaka, M. Organometallics 2000, 19, 4196. Mirzaei, F.; Han, L.-B.; Tanaka, M. Tetrahedron Lett. 2001, 42, 297.

(4) For a review see: Minami, T.; Motoyoshiya, J. Synthesis 1992, 333.

(5) Stowasser, B.; Budt, K.-H.; Jian-Qi, L.; Peyman, A.; Ruppert, D. Tetrahedron Lett. 1992, 33, 6625. Hirschmann, R.; Smith, A. B.; Taylor, C. M.; Benkovic, P. A.; Taylor, S. D.; Yager, K. M.; Sprengeler, P. A.; Benkovic, S. J. Science 1994, 265, 234. Wester, R. T.; Chambers, R. J.; Green, M. D.; Murphy, W. R. Bioorg. Med. Chem. Lett. 1994, 4, 2005.

(6) A wide variety of reactions have been performed on vinylphosphonate alkenes: Conjugate additions: Afarinkia, K.; Binch, H. M.; De Pascale, E. D. Synlett 2000, 1769. Afarinkia, K.; Binch, H. M.; Forristal, I. Synlett 2000, 1771. Cuprate additions: Li, N.-S.; Yu, S.; Kabalka, G. W. Organometallics 1999, 18, 1811. Aminohydroxylation: Thomas, A. A.; Sharpless, K. B. J. Org. Chem. 1999, 64, 8379. Cycloadditions: Ruder, S. M.; Ding, M. J. Chem. Soc., Perkin Trans. 1 2000, 1771. McClure, C. K.; Hansen, K. B.; Herzog, K. J.; Link, J. S.; Arnold, F. P. Phosphorus Sulfur 1996, 109-110, 333. Hydroboration: Pergament, I.; Srebnik, M. Org. Lett. 2001, 3, 217. Asymmetric hydrogenation: Burk, M. J.; Stammers, T. A.; Straub, J. A. Org. Lett. 1999, 1, 387. Ozonolysis: Gil, J. M.; Hah, J. H.; Park, K. Y.; Oh, D. Y. Synth. Commun. 2000, 30, 789. Oxime ether synthesis: Shen, Y.; Jiang, G.-F. Synthesis 2000, 502.

(7) Shen, Y.; Jiang, G.-F. Synthesis 2000, 99. Tago, K.; Kogen, H. Org. Lett. 2000, 2, 1975.
(8) Kouno, R.; Okauchi, T.; Nakamura, M.; Ichikawa, J.; Minami, T. J. Org. Chem. 1998, 63, 6239. Kouno, R.; Tsubota, T.; Okauchi, T.; Minami, T. J. Org. Chem. 2000, 65, 4326.

(9) Probst, M. F.; Modro, A. M.; Modro, T. A. Can. J. Chem. 1997, 75, 1131. Gil, J. M.; Oh, D. Y. J. Org. Chem. 1999, 64, 2950. Zhong, P.; Huang, X.; Xiong, Z. X. Synlett 1999, 721. Otaka, A.; Mitsuyama, E.; Watanabe, H.; Tamamura, H.; Fujii, N. Chem. Commun. 2000, 1081. Braga, A. L.; Alves, E. F.; Silveira, C. C.; de Andrade, L. L. Tetrahedron Lett. 2000, 41, 161. Jang, W. B.; Oh, D. Y.; Lee, C.-W. Tetrahedron Lett. 2000, 41, 5103 .

(10) Holt, D. A.; Erb, J. M. Tetrahedron Lett. 1989, 30, 5393. Han, L.-B.; Tanaka, M. J. Am. Chem. Soc. 1996, 118, 1571. Kazankova, M. A.; Trostyanskaya, I. G.; Lutsenko, S. V.; Beletskaya, I. P. Tetrahedron Lett. 1999, 40, 569. Okauchi, T.; Yano, T.; Fukamachi, T.; Ichikawa, J.; Minami, T. Tetrahedron Lett. 1999, 40, 5337. Zhong, P.; Xiong, Z. X.; Huang, X. Synth. Commun. 2000, 30, 273. Han, L.-B.; Mirzaei, F.; Zhao, C.-Q.; Tanaka, M. J. Am. Chem. Soc. 2000, $122,5407$.

(11) Brunner, H.; de Courcy, N. L.; Genet, J.-P. Synlett 2000, 201.

(12) Representative procedure for CM: Terminal olefin $(0.75$ mmol) and diethyl vinylphosphonate from Aldrich Chem. Co., or diethyl allylphosphonate from Acros Organics, (0.51 $\mathrm{mmol}$ ) were added simultaneously via syringe to a stirring solution of 3 ( $21 \mathrm{mg}, 0.026 \mathrm{mmol}, 5.2 \mathrm{~mol} \%)$ in $\mathrm{CH}_{2} \mathrm{Cl}_{2}(2.5$ $\mathrm{mL}, 0.2 \mathrm{M}$ in phosphonate) under a nitrogen atmosphere. The flask was fitted with a condenser and refluxed under nitrogen for 12 hours. The reaction mixture was then reduced in volume to $0.5 \mathrm{~mL}$ and purified directly on a silica gel column 
$(2 \times 10 \mathrm{~cm})$, eluting with 1:1 hexane:ethyl acetate to provide cross products as viscous oils.

(13) For recent reviews: Grubbs, R. H.; Chang, S. Tetrahedron 1998, 54, 4413. Furstner, A. Angew. Chem., Int. Ed. 2000, 39, 3013. Trnka, T. M.; Grubbs, R. H. Acc. Chem. Res. 2001, 34, 18.

(14) Schrock, R. R.; Murdzek, J. S.; Bazan, G. C.; Robbins, J.; Dimare, M.; O'Regan, M. J. Am. Chem. Soc. 1990, 112, 3875. Bazan, G. C.; Khosravi, E.; Schrock, R. R.; Feast, W. J.; Gibson, V. C.; O’Regan, M. B.; Thomas, J. K.; Davis, W. M. J. Am. Chem. Soc. 1990, 112, 8378. Bazan, G. C.; Oskam, J. H.; Cho, H. N.; Park, L. Y.; Schrock, R. R. J. Am. Chem. Soc. 1991, 113, 6899-6907.

(15) Schwab, P.; France, M. B.; Ziller, J. W.; Grubbs, R. H. Angew. Chem., Int. Ed. Engl. 1995, 34, 2039. Schwab, P.; Grubbs, R. H.; Ziller, J. W. J. Am. Chem. Soc. 1996, 118, 100.

(16) Leading CM references with 1 and 2: Crowe, W. E.; Zhang, Z. J. J. Am. Chem. Soc. 1993, 115, 10998. Crowe, W. E.; Goldberg, D. R. J. Am. Chem. Soc. 1995, 117, 5162. Blackwell, H. E.; O'Leary, D. J.; Chatterjee, A. K.; Washenfelder, R. A.; Bussmann, D. A.; Grubbs, R. H. J. Am. Chem. Soc. 2000, 122, 58.

(17) Scholl, M.; Ding, S.; Lee, C. W.; Grubbs, R. H. Org. Lett. 1999, 1, 953.

(18) Chatterjee, A. K.; Grubbs, R. H. Org. Lett. 1999, 1, 1751. Chatterjee, A. K.; Morgan, J. P.; Scholl, M.; Grubbs, R. H. J. Am. Chem. Soc. 2000, 122, 3783. Morgan, J. P.; Grubbs, R. H. Org. Lett. 2000, 2, 3153. Choi, T.; Chatterjee, A. K., Grubbs, R. H. Angew. Chem., Int. Ed. 2001, in press.
(19) Using catalyst 3 and related systems: Fürstner, A.; Thiel, O. R.; Ackermann, L.; Schanz, H.-J.; Nolan, S. P. J. Org. Chem. 2000, 65, 2204. Fürstner, A.; Thiel, O. R.; Kindler, N.; Bartkowska, B. J. Org. Chem. 2000, 65, 7990. Briot, A.; Bujard, M.; Gouverneur, V.; Nolan, S. P.; Mioskowski, C. Org. Lett. 2000, 2, 1517. Smulik, J. A.; Diver, S. T.

Tetrahedron Lett. 2001, 42, 171. Rainer, J. D.; Cox, J. M.; Allwein, S. P. Tetrahedron Lett. 2001, 42, 179. Boiteau, J.-G.; Van de Weghe, P.; Eustache, J. Tetrahedron Lett. 2001, 42, 239.

(20) Bielawski, C. W.; Grubbs, R. H. Angew. Chem., Int. Ed. 2000, 39, 2903.

(21) Hanson, P. R.; Stoianova, D. S. Tetrahedron Lett. 1998, 39 , 3939. Hanson, P. R.; Stoianova, D. S. Tetrahedron Lett. 1999, 40, 3297. Stoianova, D. S.; Hanson, P. R. Org. Lett. 2000, 2 , 1769. Sprott, K. T.; Hanson, P. R. J. Org. Chem. 2000, 65, 7913.

(22) Bujard, M.; Gouverneur, V.; Mioskowski, C. J. Org. Chem. 1999, 64, 2119. Trevitt, M.; Gouverneur, V. Tetrahedron Lett. 1999, 40, 7333. Hetherington, L.; Greedy, B.; Gouverneur, V. Tetrahedron 2000, 56, 2053. Schuman, M.; Trevitt, M.; Redd, A.; Gouverneur, V. Angew. Chem., Int. Ed. 2000, 39, 2491.

(23) Kiddle, J. J.; Babler, J. H. J. Org. Chem. 1993, 58, 3572.

(24) Chatterjee, A. K.; Choi, T.; Grubbs, R. H., manuscript in preparation.

Article Identifier:

1437-2096,E;2001,0,SI,1034,1037,ftx,en;Y05601ST.pdf 